\title{
Vergleichende Aktivitätsmessungen der Enzyme des Glucuronsäure- Xylulose-Zyklus in der Leber, dem epididymalen Fettgewebe der Ratte, in einem Morris-Hepatom und einer Fibrocyten-Kultur
}

\author{
Von Ch. Petrich, H. Reinauer und S. HollmanN \\ Aus dem Institut für Pbysiologiscbe Chemie I und dem Diabetes-Forscbungsinstitut an der Universität in Düsseldorf
}

(Eingegangen am 24. Februar 1972/18. Mai 1972)

1. Die Aktivität der Enzyme NADP-L-Gulonatdehydrogenase, NAD-L-Gulonatdehydrogenasc, NADP-Xylitdehydrogenase, NADXylitdehydrogenase und Xylulokinase wurde in Leber und erstmalig im Fettgewebe (epididymales und braunes Fettgewebe), in einem Morris-Hepatom und in einer Fibrocytenkultur gemessen.

2. Die Aktivität der Enzyme des Glucuronsäure-Xylulose-Zyklus ist bezogen auf lösliches Protein im epididymalen Fettgewebe (mit Ausnahme der NADP-Xylitdehydrogenase) höher als in der Leber. Auch im braunen Fettgewebe liegt die Aktivität der untersuchten Enzyme in der gleichen Höhe wie in der Leber, wenn man die Aktivität auf lösliches Protein bezieht.

3. Die Aktivität der Enzyme des Glucuronsäure-Xylulose-Zyklus ist im Morris-Hepatom (ACI, Stamm 9121) niedriger, jedoch noch vergleichbar mit der Leber, während in Fibrocytenkulturen die untersuchten Enzyme nicht vorkommen.

4. Dịe hohe Aktivität der untersuchten Enzyme des Glucuronsäure-Xylulose-Zyklus im Fettgewebe erklärt die extrahepatische Verwertung von Xylit im normalen und diabetischen Versuchstier, wodurch die antilipolytische Wirkung im Fettgewebe erklärt wird.

\section{Comparison of the activities of the enzymes of the glucuronic acid-xylulose patbway in the liver and epididymal adipose tissue of the rat, in a Morris bepatoma and a fibrocyte culture}

1. The activities of the enzymes NADP-L-gulonate dehydrogenase, NAD-L-gulonate dehydrogenase, NADP-xylitol dehydrogenase, NAD-xylitol dehydrogenase and xylulokinase were measured in liver and, for the first time, in adipose tissue (epididymal and brown adipose tissue), in Morris hepatoma and fibrocyte culture.

2. The activities of the enzymès of the glucuronic acid-xylulose pathway, based on the soluble protein, is higher in epididymal adipose tissue (with the exception of NADP-xylitol dehydrogenase) than in the liver. In brown adipose tissue, the activities of the enzymes studied are in the same order of magnitude as in the liver when activity is based on the soluble protein.

3. The activities of the enzymes of the glucuronic acid-xylulose pathway are lower in MorRIs hepatoma (ACI, strain 9121), but still comparable to those in the liver. The enzymes could not be detected in the fibrocyte culture

4. The high activities of the studied enzymes of the glucuronic acid-xylulose pathway in adipose tissue explains the extrahepatic utilization of xylitol in normal and diabetic experimental animals and its antilipolytic activity in adipose tissue.

Untersuchungen über die Aktivität der Enzyme des Glucuronsäure-Xylulose-Zyklus (im folgenden GXZyklus genannt) sind im Fettgewebe bisher nicht durchgeführt worden. Auch für andere Gewebe liegen keine systematischen Untersuchungen vor $(1,2,8,12)$.

Hinweise auf einen funktionstüchtigen GX-Zyklus fanden WINEGrad et al. (18), sowie KANAAMI (11) durch Verwendung von radioaktiv markierter Glucose bzw. radioaktiv markiertem Xylit im isolierten Fettgewebe. Indessen konnte keine quantitative Aussage über den Umsatz im GX-Zyklus gemacht werden.

HAYDON (6) konnte die antiketogene Wirkung von Xylit in Leberschnitten von hungernden Ratten nachweisen. Auch in vivo wirkt Xylit antilipolytisch bei alloxandiabetischen Ratten (3). Nach OprTz (13) hemmt Xylit die Lipolyse in Hungerratten, die mit Noradrenalin, Glucagon, ACTH bzw. STH vorbehandelt wurden. In der Klinik wird die antilipolytische und antiketogene Wirkung von Xylit beim Diabetes mellitus therapeutisch verwendet.
Das Ziel der vorliegenden Arbeit ist es festzustellen, ob im Fettgewebe die für die Verwertung von Xylit notwendigen Enzyme in genügender Aktivität vorhanden sind, um die antilipolytische Wirkung von Xylit in der Peripherie zu erklären.

Zur Klärung dieser Fragestellung wurden folgende Enzyme untersucht:

L-Gulonat: NADP oxidoreductase, NADP-L-Gulonatdehydrogenase (EC 1.1.1.19)

L-Gulonat: NAD oxidoreductase, NAD-L-Gulonatdehydrogenase (EC 1.1.1.45)

3-Oxo-L-gulonat carboxy-lyase, $\beta$-Keto-L-Gulonatdecarboxylase (EC 4.1.1.34)

Xylitol: NADP oxidoreductase (L-xylulose-forming), NADP-Xylitdehydrogenase (EC 1.1.1.10)

Xylitol: NAD oxidoreductase (D-xylulose-forming), NAD-Xylitdehydrogenase (EC 1.1.1.9);

ATP: D-Xylulose 5-phosphotransferase, Xylulokinase (EC 2.7.1.17). 


\section{Methodik}

Reagenzien

Adenosintriphosphat, di-Natriumsalz; $\beta$-Nicotinamid-adenindinucleotid, freie Säure; Nicotinamid-adenin-dinucleotid reduziert, Natriumsalz; Nicotinamid-adenin-dinucleotid-phosphat reduziert, Natriumsalz; Triäthanolaminhydrochlorid; Tris(hydroxymethyl)aminomethan von der $\mathrm{F}_{\mathrm{a}}$. Boehringer, Mannheim. x-Gulonsäure$\gamma$-lacton von der Fa. Fluka-AG, Buchs, Basel. D-Xylose für biochemische Zwecke von der Fa. Merck AG., Darmstadt. LXylose von der Fa. Schuchardt, München.

Alle verwendeten Reagenzien waren analytisch rein.

Kalium-[6-14C]-D-Glucuronat von, "The Radiochemical Centre“, Amersham.

Die Präparation der $\mathrm{D}$ - und $\mathrm{L}$-Xylulose erfolgte in einer Modifikation nach TousTer (16). Kalium-[1-14 C]-L-Gulonat wurde aaus Kalium-[6-14 $C]-D$-Glucuronat in einem modifizierten Verfahren nach WOLFROM und ANNO (20) hergestellt.

Die untersuchten Organe waren die Leber, das epididymale und das braune Fettgewebe der Ratte. Es wurden männliche Tiere im Gewicht von 250 bis $300 \mathrm{~g}$ vom Stamm Wistar 2 verwendet.

Die Morris-Hepatome, ACI-Stamm 9231, 23. Generation, wurden uns freundlicherweise von Herrn Priv.-Doz. Dr. Schrerber, Biochemisches Institut der Universität Freiburg/Breisgau, zur Verfügung gestellt $\left.{ }^{1}\right)$. Die Uberimpfung der Tumoren erfolgte am 24. 7. 68, die Tötung der Tiere am 6. und 7.11.68.

Die Fibrocytenkulturen wurden uns dankenswerterweise vom Hygiene-Institut der Universität Düsseldorf zur Verfügung gestellt.

Die Homogenisàtion der untersuchten Gewvebe erfolgte $1 \mathrm{~min}$ bei $0^{\circ} \mathrm{C}$ mit dem Bühler-Homogenisator. Als Homogenisationsmedium diente bei der Herstellung des Frischhomogenates $0,154 \mathrm{M} \mathrm{KCl}$ mit $0,1 \mathrm{M} \mathrm{KHCO}_{3}$ auf $\mathrm{pH}=7,4$ eingestellt. Anschließend wurde $30 \mathrm{~min}$ bei $100000 \mathrm{~g}$ zentrifugiert.

Die Herstellung des Acetontrockenpulvers erfolgte nach HoLLMANN Und LAUMaNN (8).

Als Bezugsgröße für die Enzymmessungen diente beim Homogenat das Organfrischgewicht bżw. mg Protein im Homogenat. Bei der Messung im Acetontrockenpulver wurde auf.g Acetontrockenpulver bzw. g Frischgewicht bezogen.

Die Aktivitätsmessung der NADP-L-Gulonatdehydrogenase erfolgte über die Oxidation von NADPH nach IsHIKAWA und NoGUCHr (1957). NAD-L-Gulonatdehydrogenase und $\beta$-Keto-IGulonatdecarboxylase wurden in einer Reaktion nach einer eigenen Methode gemessen. Meßgröße war die Freisetzung von ${ }^{14} \mathrm{CO}_{2}$ aus [1-14 C]-L-Gulonat (22). Die Messungen der NADP- und NADXylitdehydrogenase erfolgten im Prinzip wie von Hollmann und LAUMANN (8) bzw. Touster und Montesi (17) angegeben, jedoch wurde der NADH- bzw. NADPH-Schwund als Meßgröße gewählt. Die Bestimmung der Xylulokinase erfolgte nach AsHwell (2).

\section{Ergebnisse}

Wie aus Tabelle 1 hervorgeht, sind die sechs untersuchten Enzyme des GX-Zyklus außer in der Leber auch im epididymalen und braunen Fettgewebe der Ratte vorhanden. Bezogen auf Frischgewicht findet man zwar die höchsten Aktivitäten in der Leber, aber im Fettgewebe liegen die gemessenen Aktivitäten meist in der gleichen Größenordnung.

Bezieht man die Enzymaktivitäten in den einzelnen Organen auf die lösliche Proteinmenge, so fallen die vergleichsweise hohen Aktivitäten im epididymalen Fettgewebe auf, die ausnahmslos die der Leber übertreffen. Dies ist ohne $Z$ weifel auf den unterschiedlichen Gehalt an wasserunlöslichen Bestandteilen ( $z$. B. Neutralfette, Bindegewebe) bzw. auf den unterschiedlichen Proteingehalt zurückzuführen. Der Gesamteiweißgehalt in der Leber wird mit etwa $180-200 \mathrm{~g} / \mathrm{kg}$ Feuchtgewicht, im epididymalen Fett unter $15 \mathrm{~g} / \mathrm{kg}$ Feuchtgewicht angegebén.

Die Aktivität der NADP-L-Gulonatdehydrogenase ist in den untersuchten Organen gering und liegt fast an der Grenze der Erfaßbarkeit. Noch niedriger, aber über das freigesetzte ${ }^{14} \mathrm{CO}_{2}$ gut meßbar ist die Aktivität der NAD-L-Gulonatdehydrogenase und $\beta$-Keto-L-Gulonatdecarboxylase in allen Organen, so daß in einer dieser beiden Reaktionen der limitierende Schritt in dem gemessenen Abschnitt des GX-Zyklus vermutet werden kann.

Die Enzymmuster in den untersuchten Geweben sind nicht einheitlich. In der Leber und im epididymalen Fettgewebe ist die NADP-Xylitdehydrogenase-Aktivität mit 69,4 bzw. 52,7 U/g Protein relativ hoch, während im braunen Fettgewebe eine um eine Zehnerpotenz niedrigere Aktivität gemessen wird. Insgesamt kann die Umlagerung von L-Xylulose zu D-Xylulose als ein verhältnismäßig rasch ablaufender Vorgang angesehen werden. Die Aktivität der D-Xylulokinase ist am höchsten im epididymalen Fettgewebe, niedriger in der Leber und im braunen Fettgewebe.

Tab. 1

Enzymaktivitäten des GX-Zyklus in der Leber, im epididymalen und braunen Fettgewebe und in einem Morris-Hepatom der Ratte Angabe der Meßwerte: $\bar{x} \pm s_{x} ; n=6$

\begin{tabular}{|c|c|c|c|c|c|c|c|c|}
\hline \multirow[t]{2}{*}{ Enzyme } & \multicolumn{2}{|c|}{ Leber } & \multirow{2}{*}{$\begin{array}{l}\text { Epididymales } \\
\text { mU/g } \\
\text { Frisch- } \\
\text { gewicht }\end{array}$} & \multirow{2}{*}{$\begin{array}{l}\text { Fettgewebe } \\
\text { mU/mg } \\
\text { Protein }\end{array}$} & \multirow{2}{*}{$\begin{array}{l}\text { Braunes } \mathrm{F} \\
\mathrm{mU} / \mathrm{g} \\
\text { Frisch- } \\
\text { gewicht }\end{array}$} & \multirow{2}{*}{$\begin{array}{l}\text { Fettgewebe } \\
\text { mU/mg } \\
\text { Protein }\end{array}$} & \multicolumn{2}{|c|}{ Hepatome } \\
\hline & $\begin{array}{l}\mathrm{mU} / \mathrm{g} \\
\text { Frischgewicht }\end{array}$ & $\begin{array}{l}\mathrm{mU} / \mathrm{mg} \\
\text { Protein }\end{array}$ & & & & & $\begin{array}{c}\mathrm{mU} / \mathrm{g} \\
\text { Frischgewicht }\end{array}$ & $\begin{array}{l}\mathrm{mU} / \mathrm{mg} \\
\text { Protein }\end{array}$ \\
\hline $\begin{array}{l}\text { NADP-L-Gulonatdehydro- } \\
\text { genase }\end{array}$ & $69,5 \pm 31,6$ & $0,62 \pm 0,27$ & $47,3 \pm 20,0$ & $3,12 \pm 1,68$ & $41,7 \pm 0,06$ & $1,3 \pm 0,3$ & $118,3 \pm 10,0$ & $1,36 \pm 0,19$ \\
\hline $\begin{array}{l}\text { NAD-L-Gulonatdehydro- } \\
\text { genase und } \beta \text {-Keto-L- } \\
\text { Gulonatdecarboxylase }\end{array}$ & $20,5 \pm 6,3$ & $0,16 \pm 0,05$ & $4,04 \pm 0,55$ & $0,38 \pm 0,09$ & $4,4 \pm 1,1$ & $0,16 \pm 0,03$ & $7,3 \pm 1,8$ & $0,08 \pm 0,09$ \\
\hline NADP-Xylitdehydrogenase & $9288,2 \pm 1108,3$ & $69,4 \pm 11,8$ & $610,6 \pm 98,9$ & $52,7 \pm 13.0$ & $101,9 \pm 30,0$ & $3,0 \pm 0,8$ & $4171,7 \pm 1134,5$ & $46,96 \pm 15,64$ \\
\hline NAD-Xylitdehydrogenase & $435,0 \pm 89,4$ & $4,1 \pm 0,67$ & $125,4 \pm 28,2$ & $15,2 \pm 3,4$ & $53,1 \pm 10,0$ & $2,6 \pm 0,7$ & $155,6 \pm 17,3$ & $1,79 \pm 0,31$ \\
\hline Xylulokinase & $2533,0 \pm 431,0$ & $29,9 \pm 6,8$ & $485,0 \pm 192,0$ & $55,4 \pm 18,6$ & $621,0 \pm 69,0$ & $24,4 \pm 2,5$ & $1325,0 \pm 214,4$ & $16,6 \pm 4,6$ \\
\hline
\end{tabular}

ग) Wir danken Herrn Priv.-Doz. Dr. SChreiber für die Uberlassung der Versuchstiere. 
Insgesamt weist das braune Fettgewebe in unseren Versuchen durchweg niedrigere Enzymaktivitäten als das epididymale Fettgewebe (Tab. 1) auf.

Bei der Messung der NADP- und NAD-Xylitdehydrogenase im Acetontrockenpulver findet man bei Bezugnahme auf das Organfrischgewicht durchweg niedrigete Aktivitäten als im Frischhomogenat, offenbar infolge partieller Inaktivierung der extrahierten Enzyme. Diese partielle Inaktivierung kann durch die zusätzliche Extraktion der Xylitdehydrogenasen aus dem mitochondrialen Raum nicht wettgemacht werden.

In Morris-Hepatomen konnten alle untersuchten Enzymaktivitäten nachgewiesen werden (Tab. 1). Auffallend hoch ist hier die Aktivität der NADP-L-Gulonatdehydrogenase, die sogar die in der normalen Leber gemessenen Werte übertrifft. Alle anderen Enzyme weisen nur zwischen 30 und $50 \%$ der in der normalen Leber gefundenen Aktivitäten auf.

Bei Messungen an Fibrocytenkulturen konnten keine Aktivitäten der untersuchten Enzyme nachgewiesen werden.

\section{Diskussion}

Der tägliche Substratumsatz durch den GX-Zyklus wird mit etwa $15 \mathrm{~g}$ pro Person und Tag angegeben, wie aus Berechnungen an Personen mit Pentosurie hervorgeht (9). Für diesen Substratumsatz wurde in erster Linie die Leber und die Niere verantwortlich gemacht, denn ein funktionstüchtiger GX-Zyklus ist bislang nur in diesen Säugetierorganen nachgewiesen worden. Indessen wurde dieser Abbauweg der Glucose auch in anderen Geweben vermutet und u. a. mit den morphologisch faßbaren Veränderungen der Gefäßwände 'und der Basalmembran der Nierenglomerula beim Diabetes mellitus in Zusammenhang gebracht $(15,19)$.

Über die Aktivität der Enzyme des GX-Zyklus im Fettgewebe liegen nur wenige Daten vor. Kumahara et al. (12) fanden im Fettgewebe im Gegensatz zur Leber keine NADP-Xylitdehydrogenaseaktivität. Im Fettgewebe wurde bisher lediglich die NADP-LGulonatdehydrogenase aufgefunden (14). In unseren Untersuchungen wurden in der Leber und parallel dazu im epididymalen und braunen Fettgewebe der Ratte die Aktivitäten aller für den Glucuronsäureabbau erforderlichen Enzyme gemessen.

Erstaunlicherweise liegen die Aktivitäten dieser Enzyme im epididymalen Fettgewebe in bezug auf lösliches Zellprotein fast ausnahmslos höher als in der Leber, während im braunen Fettgewebe die Aktivitäten zwar hoch sind, jedoch von den Meßwerten in der Leber meist übertroffen werden. Vergleicht man die Aktivität der verschiedenen Enzyme untereinander, so findet man sowohl in der Leber als auch im Fettgewebe und im Morris-Hepatom die niedrigste Aktivität bei der NAD-L-Gulonatdehydrogenase, wahrscheinlich jedoch bei der $\beta$-Keto-L-Gulonatdecarboxylase, denn HäNNINEN (5) findet in der Rattenleber eine 7 bis $8 \mathrm{mal}$ höhere Aktivität der NAD-L-Gulonatdehydrogenase als bei der Aktivitätsmessung beider Reaktionsschritte in diesen Untersuchungen.

Aus den Enzymaktivitätsmessungen folgt, da $B$ im epididymalen und braunen Fettgewebe ein aktiver GXZyklus vorkommt, und somit Xylit auch extrahepatisch und extrarenal verwertet werden kann. $\mathrm{Da}$ bei einem normgewichtigen Menschen von $70 \mathrm{~kg}$ Körpergewicht etwa $10 \mathrm{~kg}$ als Bau- und Depotfett vorliegen, erreicht bei überschlagsmäßiger Berechnung die Gesamtaktivität des Fettgewebes die Werte der Leber, bei Adipositas könnte der Xylitumsatz im Fettgewebe sogar überwiegen. Die antiketogene Wirkung von Xylit ist somit durch das Zusammenwirken von Leber- und Fettgewebe zu verstehen, wobei der antilipolytischen Wirkung von Xylit im Fettgewebe eine primäre Bedeutung zukommt. In diesem Sinne sind auch die Befunde von WaNG und MeNG (23) zu werten, die mit Xylit als Substrat die höchste Syntheserate von Triglyceriden im Fettgewebe fanden.

Der GX-Zyklus ist vorwiegend im Cytosol lokalisiert. Auch das Vorkommen der NAD- und NADP-abhängigen Xylitdehydrogenasen im Cytosol ist jetzt nachgewiesen worden (1). Beide Enzyme werden darüber hinaus mit geringer Aktivität auch in den Mitochondrien der Meerschweinchenleber gefunden, und zwar die NAD-Xylitdehydrogenase in der Außenmembran, die NADP-Xylitdehydrogenase in der $\mathrm{Ma}$ trix (1).

Als Beitrag zur Enzymlokalisation wurden die Aktivitäten der beiden Xylitdehydrogenasen außer im Frischhomogenat auch im Acetontrockenpulver bestimmt. Der Vergleich der Aktivitätsmessungen im Gesamthomogenat und im Acetontrockenpulver des gleichen Organs bestätigt die Befunde, wonach die Verteilung der beiden Dehydrogenasen überwiegend cytoplasmatisch ist, denn der Aufschluß der Mitochondrien steigert die Gesamtaktivität der Xylitdehydrogenasen nicht.

Den Xylitdehydrogenasen kommt eine doppelte Bedeutung (jedenfalls in der Leberżelle) zu. Die Transhydrogenierung von NADPH auf NAD innerhalb des Cytosols bildet möglicherweise den Motor und das Kopplungsglied des GX-Zyklus mit dem Pentosephosphatzyklus. Das".Vorkommen der beiden Xylitdehydrogenasen im Cytosol und in den Mitochondrien ermöglicht einen Wasserstofftransport auf Substratbasis aus dem Cytosol in die Mitochondrien.

In den Fibrocytenkulturen wurden die Enzyme des GX-Zyklus nicht gefunden. Somit sind die gemessenen Enzymaktivitäten im Fettgewebe nicht auf den Bindegewebsanteil zurückzuführen. Die nicht meßbare Aktivität in den Fibrocyten könnten allerdings auch die Folge einer Entdifferenzierung dieser Zellen und möglicherweise Ursache für die fehlende Faserbildung sein. 


\section{Literatur}

1. Arsenis, C., Maniati, Th. \& Touster, O. (1968), J. Biol. Chem. 243, 4396-4399. - 1a. Arsenis, C. \& TOUSTER, O. (1969), J. Biol. Chem. 244, 3423-3429. - 2. Ashwell, G. (1955), Meth. Enzymol. 5, 208-211. - 3. BÄssLER, K. H. \& Dreiss, G. (1963), Klin. Wochenschr. 41, 593-595. - 4. Goto, Y., Nagano, S. \& Nakamura, H. (1969), in Pentoses and Pentitols (Horecker, B. L. \& Lang, K. Hrsg.) S. 250-255, Springer Verlag, Berlin-Heidelberg-New York. - 5. Hänninen, O. (1968), Ann. Acad. Sci. Fenn. 142, 1-96. - 6. HAYDON, R. K. (1961), Biochim. Biophys. Acta 46, 598-599. - 7. HollmanN, S. \& Touster, O. (1956), J. Amer. Chem. Soc. 78, 3544-3545. - 8. HollmanN, S. \& Laumann, G. (1967), Hoppe Seyler's Z. Physiol. Chem. 348, 1073-1076. - 9. HollmaNN, S. (1961), Nicht-glykolytische Stoffwechselwege der Glucose. S. 95-100, Thieme Verlag, Stuttgart. - 10. Ishikawa, S. \& Noguchi, K. (1957), J. Biochem., Tokyo 44, 465-469. - 11. KanaAmi, T. (1967), Tonyobo 10, 97-99. - 12. Kumahara, Y., Feingold, D. S., Freedberg, I. M. \& Hiatt, H. H. (1961), J. Clin. Endo- crinol. Metab. 21, 887-894. - 13. Oprrz, K. \& Wenntz, F. (1963), Naunyn-Schmiedebergs Arch. Pharmakol. Exp. Pathol. 245, 390-395. - 14. Shrmazono, N. \& Mano, Y. (1961), Ann. N. Y. Acad. Sci. 92, 91-104. - 15. Siperstein, M. D., Norton, W., Unger, R. H. \& Madison, L. L. (1966), Trans. Ass. Physicians 79, 330-334. - 16. Toustrer, O. (1962), Methods Carbohyd. Chem. 1, 98-101. - 17. Tousster, O. \& Montesi, G. (1962), Meth. Enzymol. 5, 317-322. - 18. WInEgrad, A. I. \& Shaw, W. N. (1964), Amer. J. Physiol. 206, 165-168. - 19. Winegrad, A. I., Clements, R. S., Beisswenger, P. J. \& BurdenRusser, C. L. (1967), in l. C. (4), S. 258-273.-20. WOLFROM, M. L. \& Anno, K. (1952), J. Amer. Chem. Soc. 74, 5583-5584. - 21. Yagamata, S., Goto, Y., Ohneda, A., Anzar, M., Kawashima, S., Kikuchi, J., Chiba, M., Maruhama, Y., Yamauchi, Y., ToYota, T., Fuguhara, N. \& Sato, S. (1967), in 1. c. (4), S. 326-333. 22. Petrich, Сh. (1970), Dissertation, Düsseldorf. - 23. WANG, M. C. \& MENG, H. C. (1971), Z. Ennährungswiss., Suppl. 11, 8-16.

Priv.-Doz. Dr. H. Reinauer 4 Düsseldorf-Benrath Hospitalstr. 1

Diabetes-Forschungsinstitut 